\title{
ASPERGILOSIS PULMONAR SECUNDARIA A NEUTROPENIA INDUCIDA POR METIMAZOL: REPORTE DE UN CASO
}

\author{
Miguel E. Pinto ${ }^{1,2, a}$, Claudia Banda ${ }^{2,3, b}$, Carlos Seas ${ }^{2,3, c}$
}

\begin{abstract}
RESUMEN
Se reporta el caso de una paciente de 48 años de edad con diagnóstico reciente de enfermedad de Graves, quien acudió a emergencia por presentar fiebre, palpitaciones y dolor faríngeo. Su tratamiento regular incluía metimazol. Al ingreso, los análisis mostraron TSH suprimido, T4 libre elevado y neutropenia. La paciente fue hospitalizada, se administraron antibióticos y factor estimulante de colonia. Después de diez días de tratamiento, la paciente presentó leucocitosis, fiebre y hemoptisis. La tomografía de tórax mostró una cavidad con múltiples nódulos en el lóbulo superior derecho. Los cultivos fueron positivos a Aspergillus fumigatus y Aspergillus flavus. Se inició tratamiento con anfotericina B y luego se cambió a voriconazol, a pesar de lo cual no hubo mejoría del cuadro. La paciente falleció por falla multiorgánica.
\end{abstract}

Palabras clave: Aspergillus; Aspergilosis pulmonar; Neutropenia; Metimazol; Agentes antitiroideos (fuente: DeCS BIREME).

\section{PULMONARY ASPERGILLOSIS DUE TO METHIMAZOLE-INDUCED NEUTROPENIA: A CASE REPORT}

\begin{abstract}
A 48-year old woman with a recent diagnosis of Graves' disease arrived at the emergency room with fever, palpitations, and a sore throat. Her regular treatment included methimazole. On admission, laboratory results showed suppressed $\mathrm{TSH}$, elevated free thyroxine, and neutropenia. She was admitted and started on antibiotics and granulocyte-macrophage colony stimulating factor (gm-csf). After ten days, the patient developed leukocytosis, fever, and hemoptysis. Chest CT scan showed a lung cavity with multiple nodules in the upper right lobe. Cultures from a lung biopsy were positive for Aspergillus Fumigatus and Aspergillus Flavus. Amphotericin B was started but then switched to voriconazole, with both treatments failing to result in clinical improvement. The patient died of multi-organ failure.
\end{abstract}

Key words: Aspergillus; Pulmonary aspergillosis; Neutropenia; Methimazole; Antithyroid agents (source: MeSH NLM).

\section{INTRODUCCIÓN}

Los fármacos antitiroideos son la base del tratamiento del hipertiroidismo, especialmente en mujeres jóvenes con enfermedad de Graves (EG) ${ }^{(1)}$. En nuestro país, de este grupo de medicamentos, solo se dispone del metimazol (MMI), el cual está asociado a diversos eventos adversos, la mayoría de los cuales son leves, transitorios y dependientes de la dosis utilizada. En menos del $5 \%$, los pacientes que reciben MMI presentan reacciones cutáneas, artralgias o molestias gastrointestinales (2); sin embargo, el evento adverso más serio es la agranulocitosis, que se puede presentar en $0,35 \%$ de los pacientes ${ }^{(3)}$, y estaría mediado por la formación de autoanticuerpos contra componentes celulares de los neutrófilos ${ }^{(2,3)}$. En pacientes con agranulocitosis inducida por MMI, se han reportado infecciones graves como sepsis por Pseudomona spp ${ }^{(4,5)}$ o, en casos más raros, infecciones micóticas como mucormicosis ${ }^{(6)}$.

El Aspergillus, hongo encontrado principalmente en el suelo, puede causar diversas enfermedades pulmonares dependiendo del estado inmune del paciente ${ }^{(7)}$. En el grupo de inmunodeprimidos puede causar aspergilosis pulmonar invasiva (API), la cual es una infección micótica oportunista de diseminación hemática, generalizada y severa, asociada con alta mortalidad; especialmente en aquellos que presentan neutropenia ${ }^{(8)}$.

En el presente reporte, se describe el caso de una paciente con diagnóstico reciente de EG en tratamiento con MMI. La paciente presentó neutropenia asociada al uso de MMI, fue hospitalizada y recibió tratamiento con antibióticos, glucocorticoides y factor estimulante de

\footnotetext{
Servicio de Endocrinología, Hospital Nacional Cayetano Heredia. Lima, Perú.

Facultad de Medicina Alberto Hurtado, Universidad Peruana Cayetano Heredia. Lima, Perú.

Instituto de Medicina Tropical Alexander von Humboldt, Universidad Peruana Cayetano Heredia. Lima, Perú.

Médico endocrinólogo; ${ }^{\mathrm{b}}$ médico cirujano; ${ }^{\mathrm{c}}$ médico infectólogo
} 
colonias (GM-CSF). Durante la hospitalización desarrolló API y falleció por falla múltiple de órganos.

\section{REPORTE DE CASO}

Paciente mujer de 48 años de edad, quien acudió al servicio de Emergencia del Hospital Nacional Cayetano Heredia, por presentar fiebre de dos semanas de evolución, palpitaciones, disfagia y dolor faríngeo. Dos meses antes de su ingreso la paciente fue diagnosticada de EG e insuficiencia cardíaca; recibía tratamiento regular con $5 \mathrm{mg}$ de $\mathrm{MMl}$; furosemida, $20 \mathrm{mg} \mathrm{VO}$ dos veces al día; y propanolol, 40 mg VO tres veces al día.

Al ingreso, los signos vitales fueron: presión arterial, 110/70 mmHg; frecuencia cardíaca, 98 latidos por minuto; frecuencia respiratoria, 28 respiraciones por minuto; temperatura oral, $38^{\circ} \mathrm{C}$; e IMC de $22 \mathrm{~kg} / \mathrm{m}^{2}$. El examen físico reveló piel caliente y húmeda, candidiasis oral, bocio difuso, edema blando en ambas piernas, y tremor distal.

Los análisis de laboratorio determinaron: tirotropina (TSH), 0,7 $\mu \mathrm{lU} / \mathrm{mL}$ (VN: 0,3-5); tiroxina libre (T4 libre), 4,34 ng/dL (VN: 0,8-1,9); tiroxina total (T4 total), 15,1 $\mu \mathrm{g} / \mathrm{dL}$ (VN: 4,5-12,5); glucosa, $92 \mathrm{mg} / \mathrm{dL}$; creatinina, $0,6 \mathrm{mg} / \mathrm{dL}$; hematocrito, 26\%; plaquetas, 233000 por $\mathrm{mm}^{3}$; y leucocitos, 1100 por $\mathrm{mm}^{3}$ (10\% neutrófilos). El electrocardiograma mostró taquicardia sinusal; la radiografía de tórax, cardiomegalia con congestión pulmonar (Figura 1).

La paciente fue hospitalizada en el Servicio de Enfermedades Infecciosas, Tropicales y Dermatológicas

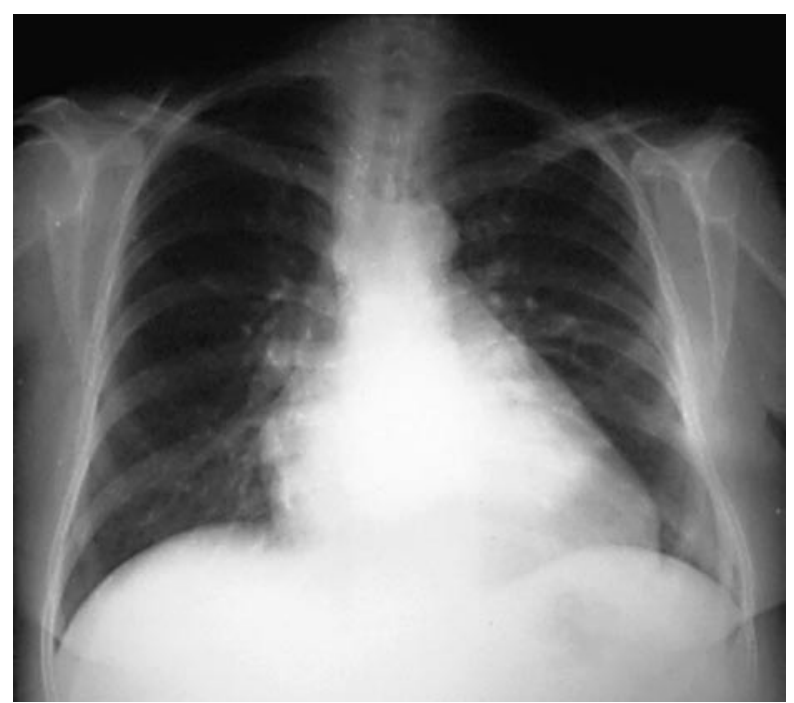

Figura 1. Radiografía de tórax al ingreso; se observa cardiomegalia y congestión pulmonar.

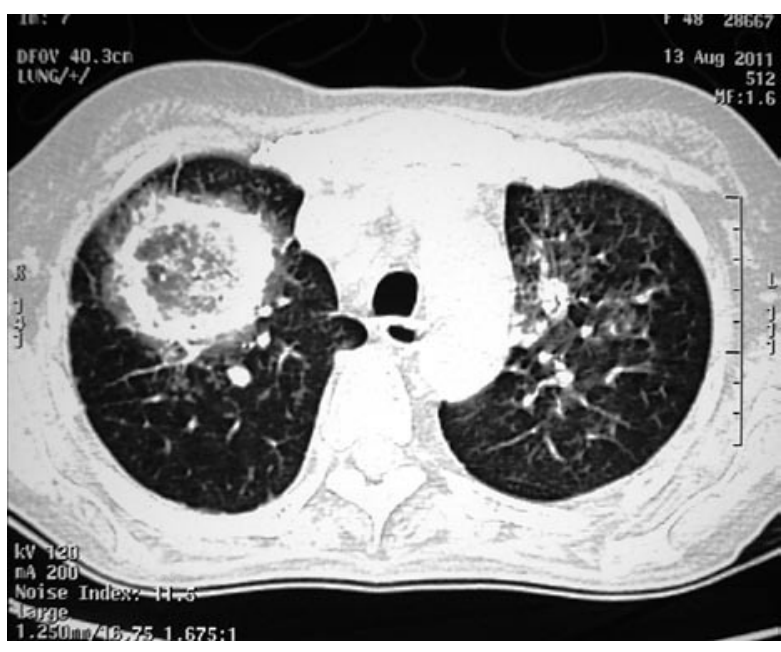

Figura 2. Tomografía de tórax después de diez días de hospitalización. Se muestra una cavidad en lóbulo superior derecho, con múltiples nódulos y signo de halo.

bajo condiciones de aislamiento invertido con el diagnóstico de neutropenia febril. Se inició tratamiento con ceftazidima EV, amikacina EV, dexametasona EV, fluconazol VO, diuréticos, beta bloqueadores y filgastrim $300 \mu \mathrm{g}$ EV dos veces al día. Después de diez días de tratamiento con filgastrim, los leucocitos se incrementaron a 82600 por $\mathrm{mm}^{3}$ (79 200 neutrófilos por $\mathrm{mm}^{3}$ ), y los controles de TSH y T4 libre fueron $0,1 \mu \mathrm{lU} / \mathrm{mL}$ y $>10 \mathrm{ng} /$ $\mathrm{dL}$, respectivamente. Sin embargo, la paciente continuaba febril, con tos, disnea y hemoptisis leve. Los antibióticos fueron cambiados a vancomicina EV y meropenem EV. Además, se agregó carbonato de litio, 300 mg VO tres veces al día, para controlar el hipertiroidismo.

La tomografía de tórax mostró una cavidad en el lóbulo superior del pulmón derecho con múltiples nódulos (Figura 2). Se realizaron exámenes de esputo y una fibrobroncoscopía con toma de muestras por lavado broncoalveolar. Los cultivos fueron positivos para Aspergillus fumigatus y Aspergillus flavus. Con estos resultados se inició tratamiento con anfotericina $\mathrm{B} E \mathrm{EV}$. Luego de ocho días de tratamiento, la paciente presentó elevación de la creatinina e hiperpotasemia, por lo que se suspendió la anfotericina $B$ y se inició tratamiento con voriconazol EV. A pesar de ello, la paciente presentó deterioro progresivo del estado general, diseminación hematógena del hongo, y falleció por falla múltiple de órganos.

\section{DISCUSIÓN}

La API fue descrita por primera vez en 1953. En los últimos años el número de casos se ha incrementado debido al mayor uso de quimioterapia y agentes 
inmunosupresores; especialmente en pacientes que han presentado una neoplasia hematológica o han recibido algún trasplante ${ }^{(9)}$. La mortalidad por API excede el $50 \%$ en pacientes neutropénicos, y es mayor a $90 \%$ en los casos de trasplante de médula ósea. La duración y gravedad de la neutropenia es el factor de riesgo más importante ${ }^{(9,10)}$. El riesgo diario de presentar API, en pacientes neutropénicos es alrededor del $1 \%$ en los primeros días, y se eleva a $4 \%$ después de la tercera semana ${ }^{(11)}$.

El ciclo infeccioso del Aspergillus empieza con la producción de conidias que son transportadas en el aire e ingresan al huésped por vía inhalatoria. En el paciente inmunocompetente, estas conidias son eliminadas por los macrófagos alveolares y los neutrófilos reclutados por una reacción inflamatoria. El riesgo de desarrollar API depende principalmente de la alteración en esta línea de defensa. Los neutrófilos son los encargados de eliminar las conidias, a través de mecanismos oxidativos y no oxidativos, e inhibir su germinación al secuestrar el hierro disponible ${ }^{(12)}$

En 3-5\% de los pacientes que reciben MMI se puede desarrollar un cuadro transitorio de granulocitopenia (<1500 granulocitos por $\mathrm{mm}^{3}$ ), que suele presentarse en los primeros 90 días de tratamiento ${ }^{(13)}$; sin embargo, en estos pacientes no se han descrito alteraciones en la función de los neutrófilos ${ }^{(2,3)}$. En los pacientes neutropénicos la API se caracteriza por crecimiento rápido del hongo, trombosis, y hemorragia. Ante la ausencia de una respuesta inflamatoria adecuada, se produce angioinvasión y diseminación hacía otros órganos, especialmente al cerebro ${ }^{(11,12)}$.

El cuadro clínico se caracteriza por fiebre persistente, tos, expectoración, disnea y hemoptisis. En algunos casos se ha descrito infartos pulmonares y dolor pleurítico por invasión vascular ${ }^{(7-9)}$. El diagnóstico es difícil y debe sospecharse en pacientes con neutropenia grave con persistencia febril y desarrollo de compromiso pulmonar. El diagnóstico histopatológico es el definitivo al hallar el hongo en cultivos positivos a Aspergillus en muestras de tejido pulmonar ${ }^{(9)}$. Sin embargo, en pacientes inmunosuprimidos, los cultivos de esputo - lavado broncoalveolar (LBA) positivos tienen un valor predictivo positivo de $80-90 \%{ }^{(7,10)}$. La radiografía de tórax muestra cambios inespecíficos tales como densidades redondeadas, infiltrados basales o cavidades ${ }^{(11)}$. La tomografía de tórax es más útil, pues muestra nódulos rodeados por el signo del "halo", que representa una zona atenuada (hemorragia) que rodea a un nódulo pulmonar ${ }^{(7,14)}$. Otra herramienta útil es la fibrobroncoscopía con LBA; especialmente en pacientes con compromiso pulmonar difuso ${ }^{(7,8)}$.
Los avances más recientes en el diagnóstico de API, están relacionados con la detección de antígenos de Aspergillus en fluidos corporales. Así se tiene el galactomanano, polisacárido de la pared celular del hongo, que es liberado durante su crecimiento y se puede detectar en sangre antes del inicio de los síntomas, aunque su rendimiento es mejor al utilizar el LBA ${ }^{(8-10)}$. También se cuenta con el beta-D-glucano, otro componente de la pared celular, que tiene una alta sensibilidad y especificidad ${ }^{(14)}$.

El tratamiento debe ser instaurado tan pronto se sospeche de API. El voriconazol es el medicamento de elección para el tratamiento ${ }^{(15)}$. La tasa de sobrevida con voriconazol es $71 \%$, en comparación con anfotericina $B$, que es de $58 \%{ }^{(11)}$. En los casos refractarios se puede utilizar como terapia de rescate al posaconazol, el caspofungin o combinaciones de antimicóticos (caspofungin, anfotericina $B$ o posaconazol) ${ }^{(11,15)}$. La resección quirúrgica esta limitada a los casos de hemoptisis masiva ${ }^{(8)}$.

En el caso presentado el diagnóstico de API fue tardío, en vista que la paciente no presentaba el antecedente de recibir quimioterapia o agentes inmunosupresores. Al realizar la revisión de la literatura científica no se encontraron reportes previos de API en pacientes con neutropenia causada por MMI. A pesar de que en años recientes el voriconazol ha surgido como la terapia de elección en casos de API, la anfotericina $\mathrm{B}$ continúa siendo el medicamento más utilizado ${ }^{(7,8)}$. En el caso referido, el uso de voriconazol se retrasó por falta de recursos económicos.

Finalmente, la terapia inmunomoduladora puede ser utilizada para disminuir el grado de inmunosupresión y como adyuvante a la terapia antimicótica. Se postula el uso de factores estimulantes de colonia ${ }^{(11)}$. En un estudio de pacientes con leucemia, quienes recibieron profilaxis con factor estimulante de colonia, se redujo la frecuencia de infecciones por hongos de 19 a 1,9\% ${ }^{(8)}$. También se postula la transfusión de granulocitos en pacientes con neutropenia refractaria a la terapia convencional, y el uso de interferón gamma ${ }^{(8,11,15)}$.

En conclusión, se reporta un caso de API en un paciente con neutropenia asociada al uso de MMI. Se debe sospechar de esta complicación en todo paciente neutropénico que persiste con fiebre, tos y hemoptisis, a pesar del tratamiento. La terapia con voriconazol debería ser iniciada ante la sospecha clínica debido a la alta mortalidad de la API.

\section{Contribuciones de autoría}

Todos los autores participaron en el análisis e interpretación de datos, revisaron en forma crítica las versiones preliminares del manuscrito y aprobaron la versión final del trabajo. 


\section{Fuentes de financiamiento}

Autofinanciado.

\section{Conflictos de interés}

Los autores declaran no tener conflictos de interés en la publicación de este artículo.

\section{REFERENCIAS BIBLIOGRÁFICAS}

1. Fumarola A, Di Fiore A, Dainelli M, Grani G, Calvanese A. Medical treatment of hyperthyroidism: state of the art. Exp Clin Endocrinol Diabetes. 2010;118(10):678-84.

2. Cooper DS. Antithyroid drugs. N Engl J Med. 2005;352(9):905-17.

3. Andersohn F, Konsen C, Garbe E. Systematic review: agranulocytosis induced by nonchemotherapy drugs. Ann Intern Med. 2007;146(9):657-65.

4. Sheng WH, Hung CC, Chen YC, Fang CT, Hsieh SM, Chang SC, et al. Antithyroid-drug-induced agranulocytosis complicated by life-threatening infections. QJM. 1999;92(8):455-61.

5. Dai WX, Zhang JD, Zhan SW, Xu BZ, Jin H, Yao Y, et al. Retrospective analysis of 18 cases of antithyroid drugs (ATD)-induced agranulocitosis. Endocr J. 2002;49(1):29-33.

6. Ramírez $\mathrm{C}$, Hernández AF, Méndez V, Trejo S, Contreras R, Espinosa de los Monteros AL, et al. Case report of mucormycosis in a patient with secondary methimazoleinduced agranulocytosis. Gac Med Mex. 2009;145(3):235-8.

7. Soubani AO, Chandrasekar PH. The clinical spectrum of pulmonary aspergillosis. Chest. 2002;121(6):1988-99.
8. Zmeili OS, Soubani AO. Pulmonary aspergillosis: a clinical update. QJM. 2007;100(6):317-34.

9. Kousha M, Tadi R, Soubani AO. Pulmonary aspergillosis: a clinical review. Eur Respir Rev. 2011;20(121):156-74.

10. Sherif R, Segal BH. Pulmonary aspergillosis: clinical presentation, diagnostic tests, management and complications. Curr Opin Pulm Med. 2010;16(3):242-50.

11. Segal BH, Walsh TJ. Current approaches to diagnosis and treatment of invasive aspergillosis. Am J Respir Crit Care Med. 2006;173(7):707-17.

12. Dagenais TR, Keller NP. Pathogenesis of Aspergillus fumigatus in invasive aspergillosis. Clin Microbiol Rev. 2009;22(3):447-65.

13. Bartalena L, Bogazzi F, Martino E. Adverse effects of thyroid hormone preparations and antithyroid drugs. Drug Saf. 1996;15(1):53-63.

14. Silveira F, Paterson DL. Pulmonary fungal infections. Curr Opin Pulm Med. 2005;1183):242-6.

15. Maertens J, Meersseman W, Van Bleyenbergh P. New therapies for fungal pneumonia. Curr Opin Infect Dis. 2009;22(2):183-90.

Correspondencia: Miguel Pinto Valdivia

Dirección: Av. Honorio Delgado 262. Lima 31, Perú.

Teléfono: (511) 4814177 / 992706110

Correo electrónico: miguelpinto72@yahoo.com

\section{Consulte la versión electrónica de la} Revista Peruana de Medicina Experimental y Salud Pública en www.pubmed.gov

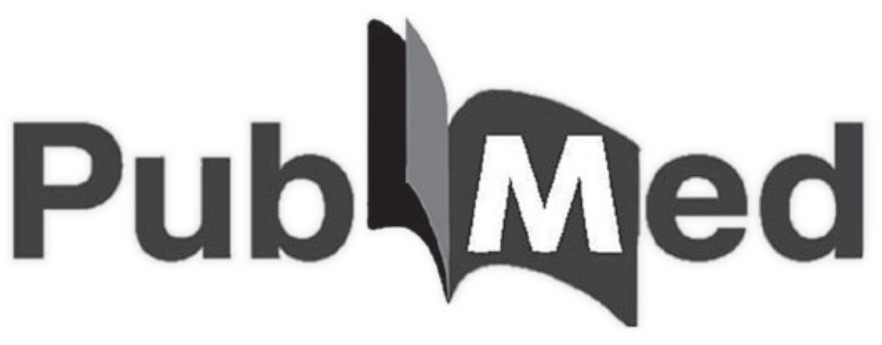

